\title{
Antenatal steroid exposure and heart rate variability in adolescents born with very low birth weight
}

\author{
Patricia A. Nixon ${ }^{1,2}$, Lisa K. Washburn², Thomas Michael O'Shea ${ }^{3}$, Hossam A. Shaltout ${ }^{4}$, Gregory B. Russell' ${ }^{5}$, Beverly M. Snively ${ }^{5}$ \\ and James C. Rose ${ }^{4}$
}

BACKGROUND: Reduced heart rate variability (HRV) suggests autonomic imbalance in the control of heart rate and is associated with unfavorable cardiometabolic outcomes. We examined whether antenatal corticosteroid (ANCS) exposure had long-term programming effects on HRV in adolescents born with very low birth weight (VLBW).

METHODS: Follow-up study of a cohort of VLBW 14-y olds born between 1992 and 1996 with 50\% exposed to ANCS. HRV in both the time and frequency domains using Nevrokard Software was determined from a 5-min electrocardiogram tracing.

RESULTS: HRV data from 89 (35 male, 53 non-black) exposed (ANCS+) and 77 (28 male, 29 non-black) unexposed (ANCS-) adolescents were analyzed. HRV did not differ between ANCS+ and ANCS- black participants. However, in non-black participants, a significant interaction between ANCS and sex was observed, with ANCS- females having significantly greater HRV than ANCS+ females and males, and ANCS- males for both time and frequency domain variables.

CONCLUSION: Among non-black adolescents born with VLBW, ANCS exposure is associated with reduced HRV with apparent sex-specificity. Reduced HRV has been associated with development of adverse cardiometabolic outcomes, thus supporting the need to monitor these outcomes in VLBW adolescents as they mature.

$\mathrm{H}$ eart rate variability (HRV) provides a noninvasive measure of cardiac autonomic modulation, reflecting heart rate control via the parasympathetic and sympathetic nervous systems (1). Low HRV is considered a marker of reduced parasympathetic control and has been associated with hypertension, diabetes, and cardiovascular mortality in adults (2), and with cardiovascular risk factors such as obesity and higher blood pressure in adolescents (3-6).

Growing evidence suggests that persons born prematurely and/or with low birth weight have increased risk for developing cardiometabolic diseases (7). The increased risk may be explained in part by Barker's fetal origins hypothesis which suggests that fetal exposures may alter developing structures and functions to promote survival in the short-term, but these programming effects may be detrimental in the long-term (8). A potential mediator of this heightened risk might be antenatal corticosteroid exposure (ANCS) which has been shown to cause decreased HRV, and subsequently, increased blood pressure in animal models (9). In humans, ANCS has been associated with alterations in fetal HRV (10), higher blood pressure in adolescence (11), and aortic stiffness and altered glucose regulation in young adulthood $(12,13)$.

The purpose of this study was therefore to examine HRV in a cohort of 14-y-old males and females born with very low birth weight (VLBW) from 1992 to 1996 which encompass the 1994 NIH Consensus Panel report promoting ANCS use for fetal lung maturation. The cohort's rate of exposure was $50 \%$, which is low when compared to current exposure rates up to $87 \%$ (14). The birth years and location also make our cohort unique with respect to racial diversity and availability of surfactant therapy as infants compared to other studies of VLBW adolescents (11) and young adults (15). As racial differences have been reported for both HRV (16) and ANCS exposure (17), we analyzed associations separately for black and non-black study participants. Furthermore, as animal models of ANCS suggest sex-specific effects $(18,19)$, we examined the potential interaction between ANCS and sex on HRV outcomes.

\section{RESULTS}

As shown in Figure 1, 193 of 479 eligible VLBW adolescents were initially recruited meeting our recruitment goal for the parent study, Prenatal Events-Postnatal Consequences (PEPC) study. After enrollment, seven participants were found to be ineligible, and one was unable to participate because of severe cerebral palsy. After further exclusions for invalid or missing data and frequent ectopic beats, the final analytic sample included 89 ANCS+ (35 male, 53 non-black) and 77 ANCS- (28 male, 29 non-black) adolescents. Demographic and neonatal characteristics were similar among eligible VLBW

\footnotetext{
'Department of Health and Exercise Science, Wake Forest University, Winston Salem, North Carolina; ${ }^{2}$ Department of Pediatrics, Wake Forest University School of Medicine, Winston Salem, North Carolina; ${ }^{3}$ Department of Pediatrics, University of North Carolina School of Medicine, Chapel Hill, North Carolina; ${ }^{4}$ Department of Obstetrics and Gynecology, Wake Forest University School of Medicine, Winston Salem, North Carolina; ${ }^{5}$ Department of Biostatistical Sciences, Wake Forest University School of Medicine, Winston Salem, North Carolina. Correspondence: Patricia A. Nixon (nixonpa@wfu.edu)

Received 11 March 2016; accepted 26 July 2016; advance online publication 5 October 2016. doi:10.1038/pr.2016.173
} 


\section{Articles $\mid$ Nixon et al.}

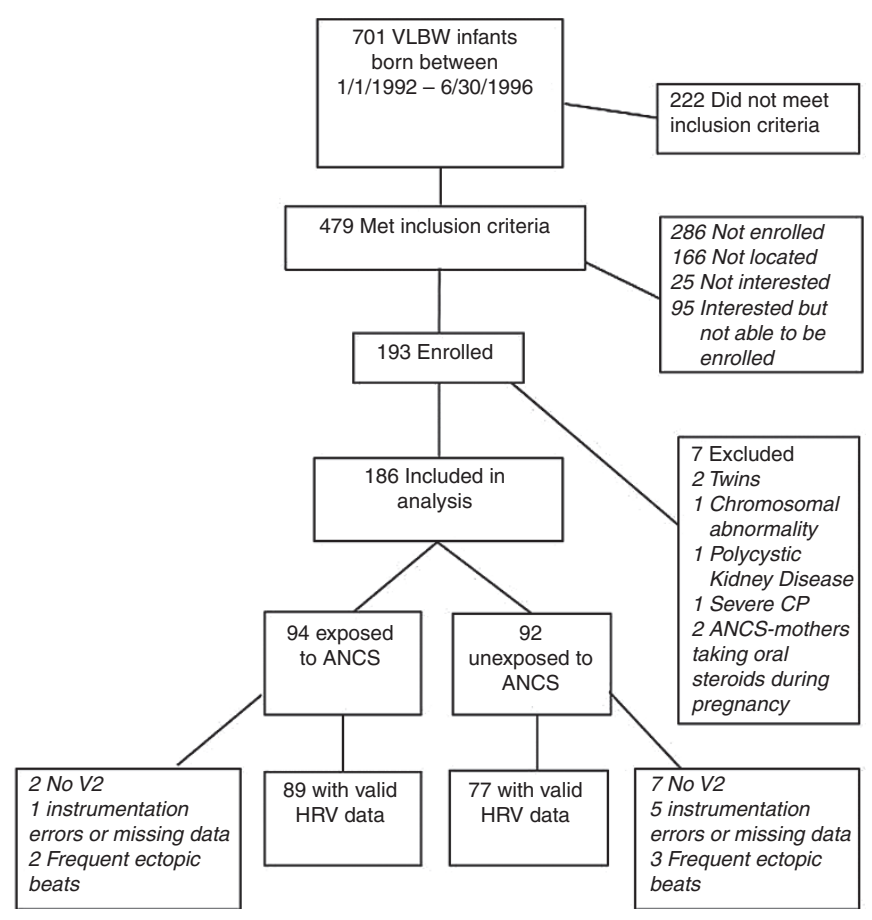

Figure 1. Follow-up details of study participants.

survivors who did or did not participate (data not shown). The neonatal and current characteristics of the remaining 166 participants are presented in Table 1. Black adolescents were less likely to have been exposed to ANCS than non-blacks (38 vs. $65 \%$ ), but birth weight parameters and gestational age did not differ between the groups. Prevalence of chronic lung disease (18 vs. $25 \%$ ), necrotizing enterocolitis (11 vs. $11 \%$ ), and major cranial ultrasound abnormalities (2 vs. 5\%) also did not differ between exposed and unexposed groups, respectively. Eightyseven of the 89 ANCS+ mothers received betamethasone, one received dexamethasone (black male offspring), and one received both betamethasone and dexamethasone (non-black female offspring). More detailed information about the number of doses and the time of dose relative to birth was available in 53 (23 M, 15 Black) of 89 ANCS-exposed women. The majority of women $(75 \%)$ received two doses (one full course), and the majority of births (72\%) occurred at least $24 \mathrm{~h}$ after, but within $7 \mathrm{~d}$, of administration of treatment. No differences in neonatal or current characteristics were found between offspring of ANCS-treated mothers with and without detailed data on exposure. At $14 \mathrm{y}$ of age, weight did not differ between groups, but ANCS+ adolescents tended to be taller and, consequently, had lower BMI Z-values on average compared to their ANCS- peers. The parental-reported prevalence of Medicaid eligibility was used as a marker of socio-economic status and did not differ between ANCS+ and ANCS- groups (37 and $43 \%$, respectively).

\section{HRV Measures}

Data of five subjects (two ANSC+) were excluded from analysis due to frequent premature ectopic beats (four ventricular and one atrial in origin). Non-normal distributions were evident for all of the HRV variables based on the Shapiro-Wilk test of normality $(P<0.05)$. Log-transformations improved the distributions of all HRV variables except pNN50 (which was not improved by $\log$ or any other standard transformation). Table 2 provides the mean HRV results by exposure for the total group, and stratified by race. For ease of presentation, untransformed means and 95\% confidence intervals (CI) are presented on the original scales of measurement, whereas the p values are based on log-transformed data, except for pNN50 which was not transformed. The analysis indicated that ANCS+ adolescents had lower HRV values for the three time domain measures as well as the frequency domain measures of $\mathrm{HF}$ and total power; however, stratification by race revealed that the exposure differences were only apparent in the non-black participants.

\section{Multiple Regression Analysis}

Multiple regression analysis was performed separately for black and non-black groups including ANCS, sex, and ANCS $\mathrm{X}$ sex as an interaction term, and adjusting for birth weight $\mathrm{Z}$-values. As shown in Figure 2a,b, significant $(P<0.05)$ ANCS X sex interactions were evident in the non-black group for variables in the both time (SDNN) (Figure 2a) and frequency (HF) (Figure 2b) domains. Post hoc comparison of LS means indicated that unexposed females had significantly greater HRV compared to exposed females and males, as well as unexposed males, with no differences among the latter three groups. A similar pattern was observed for the other time and frequency domain variables. For black adolescents, no significant differences were observed between ANCS+ and ANCSgroups for any of the HRV variables.

We also considered medications taken in the past $24 \mathrm{~h}$ that might affect HRV (such as $\beta$-agonists for asthma and stimulants for attention deficit disorder). There was no difference between ANCS groups in numbers taking these meds in the past $24 \mathrm{~h}$ (7 ANCS+ vs. 5 ANCS-), and inclusion of this information in the models did not change the results appreciably.

\section{DISCUSSION}

The results of our study suggest that ANCS exposure is associated with reduced HRV among adolescents born with VLBW. This association was limited to non-black individuals and was stronger among females than males. The finding that ANCS exposure was associated with decreased HRV is consistent with our colleagues' results in the sheep model $(9,20)$, although HRV was only studied in male sheep. An unexpected finding was the higher LF in unexposed females which may suggest greater sympathetic modulation of heart rate. However, LF is believed to reflect both sympathetic and parasympathetic control, and has been suggested to be determined by parasympathetic activity (21). Recent evidence also suggests that LF is not a measure of cardiac sympathetic tone, but more likely reflects modulation of cardiac autonomic outflows by baroreflexes, with higher LF correlating with greater cardiovagal gain (22).

Other studies have examined the effects of ANCS exposure on fetal HRV (10,23-25). As reviewed by Mulder et al. (26), administration of ANCS was generally associated with an 
Table 1. Participants' neonatal and current characteristics by exposure and race*

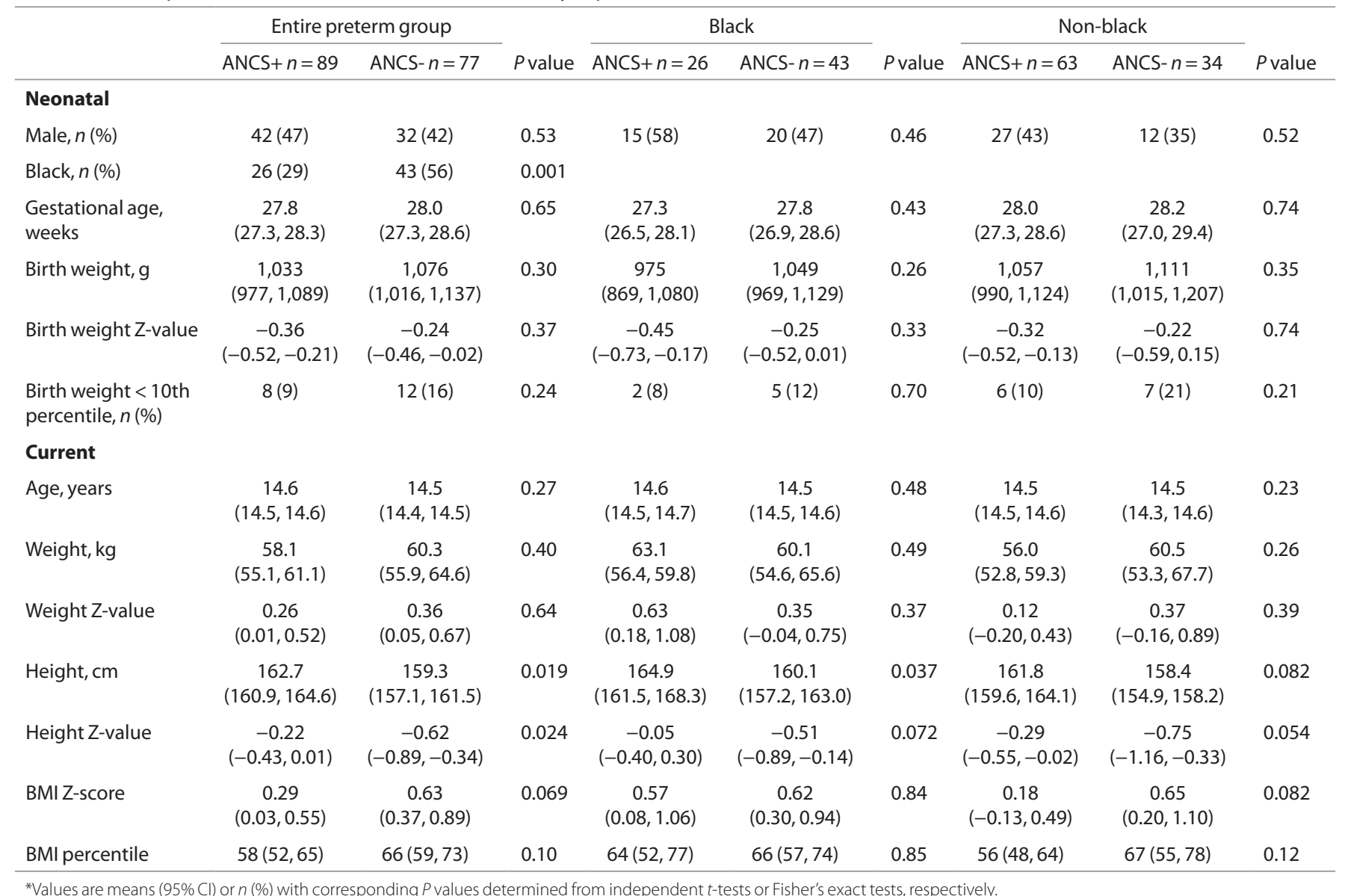

ANCS, antenatal corticosteroid.

initial increase in fetal HRV in the first $24 \mathrm{~h}$, followed by a subsequent decrease of $20-30 \%$ of pretreatment levels by $48-72 \mathrm{~h}$, and then returning to baseline levels within $4(10,23)$ to $7 \mathrm{~d}$ $(24,25)$ after cessation of treatment. In a relatively short-term follow-up study of neonates, no differences in HRV were evident $7 \mathrm{wk}$ after exposure to ANCS (27).

To our knowledge, our study is the first to report possible long-term effects of ANCS exposure on HRV in adolescents born prematurely with VLBW. The long-term effects of ANCS on HRV may reflect programming during critical periods of fetal development. Evidence suggests that the development of cardiac autonomic innervation extends into early postnatal life and displays considerable plasticity (28). Bian and colleagues $(29,30)$ have shown in rats that fetal ANCS exposure (with dexamethasone) alters development of cardiac noradrenergic and sympathetic processes including enhanced cardiac adenylate cyclase reactivity to $\beta$-adrenergic and nonadrenergic stimuli. Consequently, exposure to ANCS during the critical period of autonomic maturation may have led to alterations of autonomic control of heart rate in our adolescents.

Sex differences in HRV have been reported in children and adolescents. Results from larger studies $(31,32)$ indicate that boys exhibit greater HRV than girls when examining time domain variables and the frequency domain variable HF. In contrast, we found higher HRV in females than males, specifically among those who were not exposed to ANCS. There is evidence supporting sex differences for other outcomes such as blood pressure, markers of the renin-angiotensin system, and sympathoadrenal function, with females generally exhibiting more favorable outcomes $(33,34)$. Our colleagues have also reported sex differences in ANCS programming effects on renal function in the sheep model, with some of the differences being age-dependent, possibly mediated via changes in sex steroid hormones with maturation $(18,19)$.

Racial differences have also been reported for HRV (16) and fetal programming (35), but no study has examined race as a potential effect modifier of ANCS on HRV. Higher HRV, among black adolescents, as observed in our study as well as a study of healthy adolescents (16), should reduce risk for cardiometabolic disease. Assuming our finding of higher HRV among black VLBW adolescents is generalizable, the higher risk of cardiometabolic disease generally observed among black individuals is more likely attributable to other factors such as peripheral vasoconstriction or renal dysfunction.

There are several limitations to our study. The study design is observational and therefore not a randomized controlled trial of ANCS exposure. However, with the proven beneficial effects of ANCS on respiratory morbidity and survival 


\section{Articles $\mid$ Nixon et al.}

Table 2. Comparison of HRV variables by ANCS and within race*

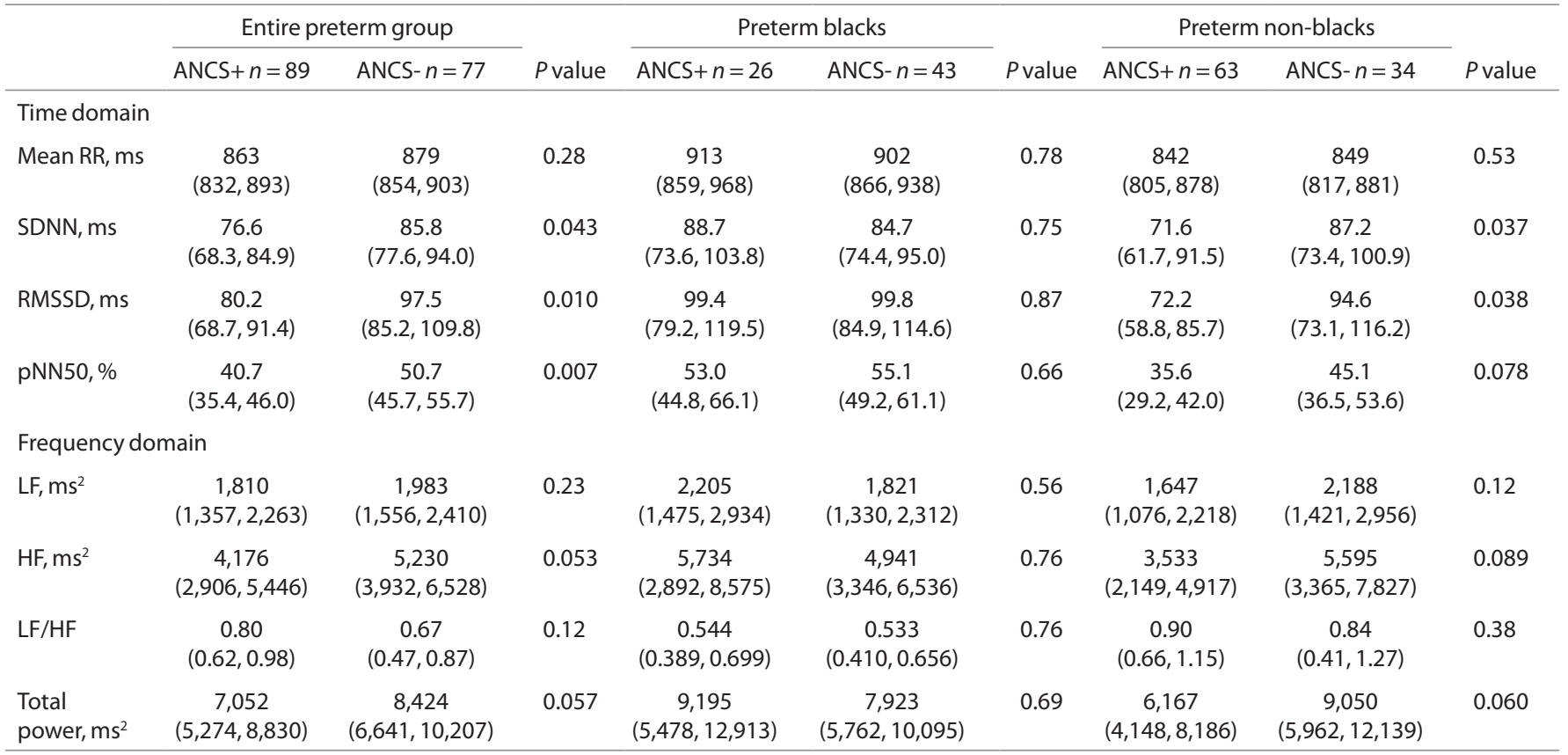

*Values presented are means ( $95 \%$ Cl) for untransformed variables, but p value is from independent t-tests on transformed variables (except pNN50). ANCS, antenatal corticosteroid; Mean RR, mean R-R interval; SDNN, SD of all N to N intervals; RMSSD, square root of the mean of sum of squares of differences in successive N to N intervals; pNN50: percent of successive $\mathrm{N}$ to $\mathrm{N}$ intervals differing by at least $50 \mathrm{~ms}$; LF, low frequency power; $\mathrm{HF}$, high frequency power; LF/HF, ratio of LF to HF power.
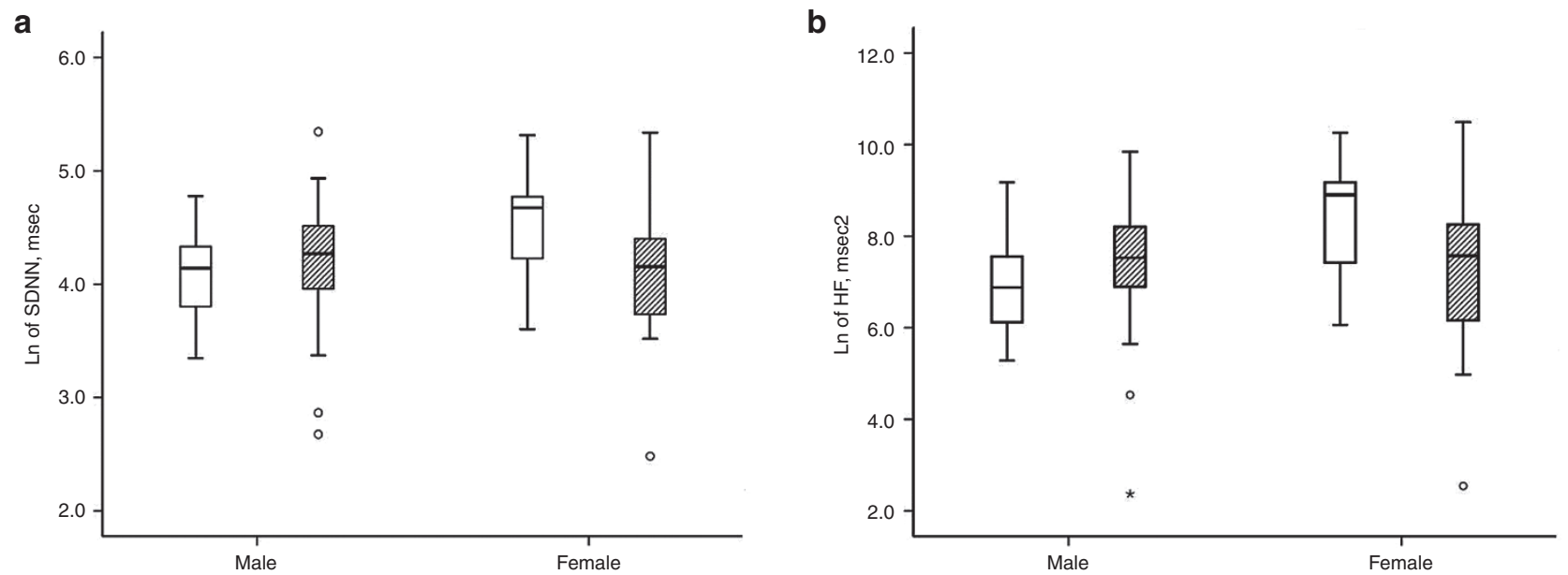

Figure 2. HRV in nonblack males and females by ANCS exposure. (a, b) Boxplots demonstrating a significant interaction between antenatal corticosteroid (ANCS) $\times$ sex from multiple regression analysis in non-black participants for: (a) the log-transformed time domain variable of SD of all $\mathrm{N}$ to $\mathrm{N}$ intervals (SDNN) $(P=0.043)$, and $(\mathbf{b})$ the log-transformed frequency domain variable of high frequency power $(\mathrm{HF})(P=0.017)$. ANCS+ and ANCS- are represented by hatched and open boxes, respectively. The regression models included ANCS, sex, ANCS $\times$ sex, and birth weight z-value. Post-hoc comparisons of least square means indicated that ANCS- females had significantly greater SDNN and HF compared to ANCS+ females and males as well as ANCS- males.

in premature infants (36), treatment with ANCS for preterm labor has become standard practice with up to $87 \%$ of VLBW infants currently exposed (14), and conducting a randomized controlled trial would be unethical. The $50 \%$ exposure rate of our sample (primarily due to birth years surrounding the 1994 NIH Consensus Panel promoting ANCS use) provides us with a unique study sample to examine the long-term effects of ANCS exposure which cannot be replicated in view of current exposure rates. Furthermore, the birth years and location of our cohort make the results more generalizable to a racially diverse current VLBW population born in the postsurfactant era.

We also lacked complete data on the number of doses and timing for all of our ANCS- exposed participants. In view of the high exposure rates that exist today, future studies should examine the effects of single vs. multiple ANCS courses on HRV as well as the timing of exposure relative to birth. It would also be interesting to see if the ANCS exposure differences are evident in children who were carried to term. Finally, there are many other early life exposures (in utero and perinatal) as well as other 
factors (e.g., menstrual cycle phase, aerobic fitness) which may influence HRV but were not considered in our study.

In summary, our study results suggest that ANCS exposure is associated with reduced HRV in adolescents with apparent race- and sex-specificity. Reduced HRV has been associated with development of adverse cardiometabolic outcomes, supporting the need to closely follow this at-risk population as they mature. The sex- and race-specific differences associated with ANCS exposure underscore the importance of considering these factors as possible modifiers of the presumed programming effect of ANCS on physiologic and health outcomes.

\section{METHODS}

The study was approved by the Institutional Review Boards of Wake Forest Baptist and Forsyth Medical Centers. Written informed consent was obtained from a parent/legal guardian and assent was obtained from the adolescent.

Participants were recruited from a cohort of 479 infants born with VLBW $(<1,500 \mathrm{~g})$ between 1992 and 1996 at a regional perinatal center (Forsyth Medical Center) who also met the following criteria: $14 \mathrm{y}$ of age, singleton birth without a major congenital anomaly, and clinical evaluation at 1-y adjusted age. Participants were asked to report for three study visits as part of the Prenatal Exposure Postnatal Consequences (PEPC) study and were paid a total of \$225 for all three study visits. Parents were also paid $\$ 25$ for each visit to cover travel expenses.

\section{HRV Measurement}

Heart rate variability was measured at the second study visit between noon and $2 \mathrm{pm}$ in a quiet examination room of the clinical research unit in accordance with standardized guidelines (1). Participants were asked to refrain from eating in the previous $4 \mathrm{~h}$ or exercising the morning of the visit. The participant was prepped with three electrodes (Lead II configuration) that were connected to a Biopac MP36 (Biopac Systems, Goleta, CA) for continuous recording of the electrocardiogram (ECG), and then asked to lie quietly on a bed for $10 \mathrm{~min}$. The first $5 \mathrm{~min}$ were used to familiarize the participant to the environment, and data from the subsequent 5 min were analyzed using Nevrokard-HRV Software (Slovenia) to identify $\mathrm{R}$ waves from the ECG and subsequent determination of HRV in both time and frequency domains (1). The primary time domain variables of interest were the standard deviation of all $\mathrm{R}$ to $\mathrm{R}$ intervals, commonly reported as $\mathrm{N}$ to $\mathrm{N}$ intervals (SDNN), the square root of the mean of sum of squares of differences in successive $\mathrm{N}$ to $\mathrm{N}$ intervals (rMSSD), and the percent of successive $\mathrm{N}$ to $\mathrm{N}$ intervals that differed by at least $50 \mathrm{~ms}$ (pNN50). For all of these variables, a higher value suggests greater HRV. The primary frequency domain variables of interest were low frequency power (LF), and high frequency power (HF), the ratio of LF to $\mathrm{HF}$ (LF/HF), and the total power determined from power spectral analysis using fast Fourier transformations (Hamming window) with band widths 0.04 to $0.15 \mathrm{~Hz}$ and 0.15 to $0.40 \mathrm{~Hz}$ for LF and HF, respectively. The HF is believed to reflect parasympathetic modulation of heart rate. Although controversial (37), the LF and the LF/HF are commonly used to reflect sympathetic regulation and sympathovagal balance, respectively. Consequently, higher HF and total power, and lower LF and LF/HF, are desirable suggesting greater parasympathetic control of heart rate. Participants with ECG tracings exhibiting frequent ( $>5$ per minute) premature ventricular or atrial contractions (PVCs and PACs respectively) were excluded. Those with infrequent PVCs or PACs ( $\sim 5$ or less during the $5 \mathrm{~min}$ period) were included in the analysis but the ECG tracings were edited by deleting the segment of time from the peak of the $\mathrm{R}$ wave for the QRS complex occurring one beat before the ectopic beat to the peak of the R wave for the QRS complex occurring two beats following the ectopic beat (38).

\section{Neonatal Characteristics}

Neonatal characteristics including birth weight, gestational age, prevalence of neonatal illness, and ANCS exposure (including date and dose of ANCS when available) were obtained from a research database as well as maternal and participant medical records by a research nurse. Gestational age was determined in order of availability from either first trimester ultrasound, maternal report of last menstrual period, or, lastly, clinical assessment of the newborn infant. Birth weight $\mathrm{z}$-values and percentiles was determined from gestational ageand gender-specific reference data as a marker of fetal growth (39). A diagnosis of chronic lung disease (CLD) was based on supplemental oxygen requirement at $36 \mathrm{wk}$ postmenstrual age (40). A diagnosis of necrotizing enterocolitis (NEC) was based on Bell's criteria with stage $\geq 2$ (41). A major cranial ultrasound abnormality was defined as previously described (42).

\section{Current Characteristics}

Height and weight were measured in triplicate (light clothing without shoes) using a wall-mounted stadiometer and digital platform scale respectively. Body mass index (BMI) was calculated from the ratio of weight in $\mathrm{kg}$ to height in $\mathrm{m}^{2}$, and age- and gender-specific percentiles were determined (43). Race was determined by questionnaire completed by the participant's parent/guardian. Parental report of Medicaid eligibility was used as a marker of socio-economic status. Medications (including over-the-counter) taken on a regular basis and in the past $24 \mathrm{~h}$ were reported by both child and parent.

\section{Data Analysis}

Data were analyzed using SAS version 9.4. Measures of central tendency and dispersion were examined, and log transformations were applied to HRV variables with Shapiro-Wilk test of normality results having a $P<0.05$ to improve distributional characteristics prior to analyses. Data are presented as means and their corresponding 95\% confidence intervals (CI) or $n(\%)$. In view of racial differences in HRV and exposure to ANCS, we decided a priori to stratify results by race (black and non-black). Between-group (ANCS+ vs. ANCS-) differences were then examined using independent samples t-tests for continuous variables and Fisher's exact tests for categorical variables. We examined the potential interaction of ANCS with sex by entering the product term $($ ANCS $\times$ sex) into multiple regression models. Birth weight $\mathrm{z}$-value (a marker of fetal growth) was added to regression models to adjust for potential confounding effects. Post hoc pairwise comparisons of least squares (LS) means (generated by the SAS GLM procedure) were used to identify differences among the four ANCS X sex group combinations.

\section{ACKNOWLEDGMENTS}

We wish to acknowledge Alice Scott, RN and Patti Brown, RN, and the Clinical Research Unit nurses for their assistance in coordinating and collecting data, the undergraduate and graduate students from the HES Department of Wake Forest University who assisted with data collection, and the participants and their parents for their time and dedication to the project.

\section{STATEMENT OF FINANCIAL SUPPORT}

The Eunice Kennedy Shriver National Institute of Child Health and Human Development (grant PO1HD0474584), the General Clinical Research Center of Wake Forest University Baptist Medical Center (grant MO1 RR07122), and the Intramural Research Support Committee of Wake Forest Medical School and the Brenner Center for Child and Adolescent Health.

Disclosure: The authors have no conflicts of interest.

\section{REFERENCES}

1. Heart rate variability. Standards of measurement, physiological interpretation, and clinical use. Task Force of the European Society of Cardiology and the North American Society of Pacing and Electrophysiology. Eur Heart J 1996;17:354-81.

2. Wulsin LR, Horn PS, Perry JL, Massaro JM, D’Agostino RB. Autonomic imbalance as a predictor of metabolic risks, cardiovascular disease, diabetes, and mortality. J Clin Endocrinol Metab 2015;100:2443-8.

3. Farah BQ, Barros MV, Balagopal B, Ritti-Dias RM. Heart rate variability and cardiovascular risk factors in adolescent boys. J Pediatr 2014;165:945-50.

4. Rodríguez-Colón SM, He F, Bixler EO, et al. Metabolic syndrome burden in apparently healthy adolescents is adversely associated with cardiac autonomic modulation-Penn State Children Cohort. Metabolism 2015;64:626-32. 


\section{Articles Nixon et al.}

5. Rodríguez-Colón S, He F, Bixler EO, et al. The circadian pattern of cardiac autonomic modulation and obesity in adolescents. Clin Auton Res 2014;24:265-73.

6. Ryder JR, O'Connell M, Bosch TA, et al. Impaired cardiac autonomic nervous system function is associated with pediatric hypertension independent of adiposity. Pediatr Res 2016;79:49-54.

7. Parkinson JR, Hyde MJ, Gale C, Santhakumaran S, Modi N. Preterm birth and the metabolic syndrome in adult life: a systematic review and metaanalysis. Pediatrics 2013;131:e1240-63.

8. Barker DJ. The origins of the developmental origins theory. J Intern Med 2007;261:412-7.

9. Shaltout HA, Rose JC, Chappell MC, Diz DI. Angiotensin-(1-7) deficiency and baroreflex impairment precede the antenatal Betamethasone exposure-induced elevation in blood pressure. Hypertension 2012;59:453-8.

10. Mulder EJ, Derks JB, Visser GH. Antenatal corticosteroid therapy and fetal behaviour: a randomised study of the effects of betamethasone and dexamethasone. Br J Obstet Gynaecol 1997;104:1239-47.

11. Doyle LW, Ford GW, Davis NM, Callanan C. Antenatal corticosteroid therapy and blood pressure at 14 years of age in preterm children. Clin Sci (Lond) 2000;98:137-42.

12. Kelly BA, Lewandowski AJ, Worton SA, et al. Antenatal glucocorticoid exposure and long-term alterations in aortic function and glucose metabolism. Pediatrics 2012;129:e1282-90.

13. Dalziel SR, Walker NK, Parag V, et al. Cardiovascular risk factors after antenatal exposure to betamethasone: 30-year follow-up of a randomised controlled trial. Lancet 2005;365:1856-62.

14. Stoll BJ, Hansen NI, Bell EF, et al.; Eunice Kennedy Shriver National Institute of Child Health and Human Development Neonatal Research Network. Trends in care practices, morbidity, and mortality of extremely preterm neonates, 1993-2012. JAMA 2015;314:1039-51.

15. Hovi P, Andersson S, Eriksson JG, et al. Glucose regulation in young adults with very low birth weight. N Engl J Med 2007;356:2053-63.

16. Wang X, Thayer JF, Treiber F, Snieder H. Ethnic differences and heritability of heart rate variability in African- and European American youth. Am J Cardiol 2005;96:1166-72.

17. Carlo WA, McDonald SA, Fanaroff AA, et al.; Eunice Kennedy Shriver National Institute of Child Health and Human Development Neonatal Research Network. Association of antenatal corticosteroids with mortality and neurodevelopmental outcomes among infants born at 22 to 25 weeks' gestation. JAMA 2011;306:2348-58.

18. Tang L, Bi J, Valego N, et al. Prenatal betamethasone exposure alters renal function in immature sheep: sex differences in effects. Am J Physiol Regul Integr Comp Physiol 2010;299:R793-803.

19. Su Y, Bi J, Pulgar VM, Figueroa J, Chappell M, Rose JC. Antenatal glucocorticoid treatment alters $\mathrm{Na}+$ uptake in renal proximal tubule cells from adult offspring in a sex-specific manner. Am J Physiol Renal Physiol 2015;308:F1268-75.

20. Shaltout HA, Rose JC, Figueroa JP, Chappell MC, Diz DI, Averill DB. Acute AT(1)-receptor blockade reverses the hemodynamic and baroreflex impairment in adult sheep exposed to antenatal betamethasone. Am J Physiol Heart Circ Physiol 2010;299:H541-7.

21. Reyes del Paso GA, Langewitz W, Mulder LJ, van Roon A, Duschek S. The utility of low frequency heart rate variability as an index of sympathetic cardiac tone: a review with emphasis on a reanalysis of previous studies. Psychophysiology 2013;50:477-87.

22. Goldstein DS, Bentho O, Park MY, Sharabi Y. Low-frequency power of heart rate variability is not a measure of cardiac sympathetic tone but may be a measure of modulation of cardiac autonomic outflows by baroreflexes. Exp Physiol 2011;96:1255-61.

23. Subtil D, Tiberghien P, Devos P, et al. Immediate and delayed effects of antenatal corticosteroids on fetal heart rate: a randomized trial that com- pares betamethasone acetate and phosphate, betamethasone phosphate, and dexamethasone. Am J Obstet Gynecol 2003;188:524-31.

24. Senat MV, Minoui S, Multon O, Fernandez H, Frydman R, Ville Y. Effect of dexamethasone and betamethasone on fetal heart rate variability in preterm labour: a randomised study. Br J Obstet Gynaecol 1998;105:749-55.

25. Multon O, Senat MV, Minoui S, Hue MV, Frydman R, Ville Y. Effect of antenatal betamethasone and dexamethasone administration on fetal heart rate variability in growth-retarded fetuses. Fetal Diagn Ther 1997;12: 170-7.

26. Mulder EJ, de Heus R, Visser GH. Antenatal corticosteroid therapy: shortterm effects on fetal behaviour and haemodynamics. Semin Fetal Neonatal Med 2009;14:151-6.

27. Schäffer L, Burkhardt T, Tomaske M, et al. Effect of antenatal betamethasone administration on neonatal cardiac autonomic balance. Pediatr Res 2010;68:286-91.

28. Hasan W. Autonomic cardiac innervation: development and adult plasticity. Organogenesis 2013;9:176-93.

29. Bian X, Seidler FJ, Slotkin TA. Fetal dexamethasone exposure interferes with establishment of cardiac noradrenergic innervation and sympathetic activity. Teratology 1993;47:109-17.

30. Bian XP, Seidler FJ, Slotkin TA. Promotional role for glucocorticoids in the development of intracellular signalling: enhanced cardiac and renal adenylate cyclase reactivity to beta-adrenergic and non-adrenergic stimuli after low-dose fetal dexamethasone exposure. J Dev Physiol 1991;16: 331-9.

31. Michels N, Clays E, De Buyzere M, et al. Determinants and reference values of short-term heart rate variability in children. Eur J Appl Physiol 2013;113:1477-88.

32. Jarrin DC, McGrath JJ, Poirier P, et al. Short-term heart rate variability in a population-based sample of 10-year-old children. Pediatr Cardiol 2015;36:41-8.

33. Gabory A, Attig L, Junien C. Sexual dimorphism in environmental epigenetic programming. Mol Cell Endocrinol 2009;304:8-18.

34. Tomat AL, Salazar FJ. Mechanisms involved in developmental programming of hypertension and renal diseases. Gender differences. Horm Mol Biol Clin Investig 2014;18:63-77.

35. Mzayek F, Sherwin R, Fonseca V, et al. Differential association of birth weight with cardiovascular risk variables in African-Americans and Whites: the Bogalusa heart study. Ann Epidemiol 2004;14:258-64.

36. Roberts D, Dalziel S. Antenatal corticosteroids for accelerating fetal lung maturation for women at risk of preterm birth. Cochrane Database Syst Rev Online 2006;3:CD004454.

37. Parati G, Mancia G, Di Rienzo M, Castiglioni P. Point: cardiovascular variability is/is not an index of autonomic control of circulation. J Appl Physiol 2006;101:676-682.

38. Peltola MA. Role of editing of R-R intervals in the analysis of heart rate variability. Front Physiol 2012;3:148.

39. Oken E, Kleinman KP, Rich-Edwards J, Gillman MW. A nearly continuous measure of birth weight for gestational age using a United States national reference. BMC Pediatr 2003;3:6.

40. Shennan AT, Dunn MS, Ohlsson A, Lennox K, Hoskins EM. Abnormal pulmonary outcomes in premature infants: prediction from oxygen requirement in the neonatal period. Pediatrics 1988;82:527-32.

41. Walsh MC, Kliegman RM. Necrotizing enterocolitis: treatment based on staging criteria. Pediatr Clin North Am 1986;33:179-201.

42. Washburn LK, Dillard RG, Goldstein DJ, Klinepeter KL, deRegnier RA, O'Shea TM. Survival and major neurodevelopmental impairment in extremely low gestational age newborns born 1990-2000: a retrospective cohort study. BMC Pediatr 2007;7:20.

43. Kuczmarski RJ, Ogden CL, Grummer-Strawn LM, et al. CDC growth charts: United States. Adv Data 2000;1-27. 